\title{
CONHECIMENTO SOBRE ALIMENTAÇÃO PARA O TRATAMENTO DE DIABETES MELLITUS
}

\author{
KNOWLEDGE ABOUT FOOD FOR THE TREATMENT OF DIABETES \\ MELLITUS
}

Cláudia de Sousa Freitas ${ }^{1}$ Lidiane Oliveira Duarte ${ }^{2}$

Larissa de Brito Medeiros ${ }^{3}$

RESUMO: Objetivo: Avaliar o nível de conhecimento do paciente com Diabetes Mellitus (DM) tipo 2 sobre a alimentação adequada para o tratamento da doença e relacioná-lo com condições socioeconômicas, controle glicêmico e estado nutricional. Metodologia: Trata-se de uma pesquisa transversal com abordagem quantitativa, realizada em duas Unidades de Saúde da Família (USF) do município de Uiraúna-PB. A amostra foi composta por 30 participantes com diagnóstico de DM2 de ambos os sexos, maiores de 45 anos, frequentadores do Grupo de Hipertensos e Diabéticos (Hiperdia) que fazem acompanhamento com as equipes das USFs. Para avaliação do conhecimento dos pacientes diabéticos sobre a alimentação adequada para controle do DM2, foi aplicado um questionário com 16 questões. Além disso, dados sobre condições socioeconômicas, prática de atividade física e estado nutricional também foram coletados. O teste de glicemia capilar foi realizado no dia do encontro com o grupo de Hiperdia. Os dados obtidos foram tabulados no programa Microsoft Office Excelß 2016 e a análise estatística foi realizada pelo software Sigmastat 3.5. Para avaliação do conhecimento dos participantes, foram considerados "baixo conhecimento" um total de acertos $\leq 50 \%$ das questões, "conhecimento suficiente" um percentual de acertos $\geq 70 \%$ e "conhecimento regular" o percentual de acertos entre 51 e 69\%. Resultados: A partir da análise dos questionários aplicados, metade dos participantes $(50 \%)$ apresentaram baixo conhecimento e 36,67\% conhecimento regular sobre alimentação adequada para o DM. Em relação aos fatores socioeconômicos, $93,33 \%$ dos participantes possui baixa renda familiar e mais da metade $(53,33 \%)$ são analfabetos. Dos 30 indivíduos estudados, 43,33\% estão acima do peso e, apesar da mesma quantidade de participantes (43,33\%) estarem com o peso adequado, nota-se prevalência de adiposidade central na maioria dos indivíduos, a partir dos altos valores de circunferência de cintura $(93,33 \%)$ e razão cintura-quadril (83,33\%). Grande parte dos pacientes do estudo (76,66\%) possui a glicemia acima das recomendações de controle glicêmico da Sociedade Brasileira de Diabetes.

\footnotetext{
${ }_{1}^{1}$ Pós-Graduanda em Nutrição Clínica Funcional da Faculdade Santa Maria.

${ }^{2}$ Pós-Graduanda em Nutrição Clínica Funcional da Faculdade Santa Maria.

${ }^{3}$ Docente do Curso de Nutrição da Faculdade Santa Maria.
} 
Conclusão: Apesar das limitações, esse estudo mostra como fatores socioeconômicos podem interferir no conhecimento sobre alimentação adequada para o DM e, por conseguinte, no estado nutricional e controle glicêmico dos participantes. Com isso, sugere-se a realização de mais pesquisas na região estudada, com maior número de participantes e de variáveis para corroborar com os resultados encontrados e proporcionar ações educativas a nível populacional mais eficazes sobre a importância da alimentação adequada como parte imprescindível do tratamento.

Palavras chave: Diabetes Mellitus tipo 2. Dieta. Estado nutricional. Fatores Socioeconômicos. Glicemia.

ABSTRACT: Objective: To assess the level of knowledge of the type 2 Diabetes Mellitus (DM) patient about adequate food for the treatment of the disease and relate it to socioeconomic conditions, glycemic control and nutritional status. Methodology: This is a cross-sectional study with a quantitative approach, carried out in two Family Health Units (USF) in the city of Uiraúna-PB. The sample consisted of 30 participants diagnosed with DM2 of both sexes, over 45 years of age, who frequent the Hypertensive and Diabetic Group (Hiperdia) who are followed up with the FHU teams. To assess the knowledge of diabetic patients about adequate nutrition to control DM2, a questionnaire with 16 questions was applied. In addition, data on socioeconomic conditions, physical activity and nutritional status were also collected. The capillary blood glucose test was performed on the day of the meeting with the group of Hiperdia. The data obtained were tabulated in the Microsoft Office ExcelB 2016 program and the statistical analysis was performed using the Sigmastat 3.5 software. To assess the participants' knowledge, a total of correct answers $\leq 50 \%$ of the questions was considered "low knowledge", "sufficient knowledge" a percentage of correct answers $\geq 70 \%$ and "regular knowledge" the percentage of correct answers between 51 and 69\%. Results: From the analysis of the applied questionnaires, half of the participants (50\%) had low knowledge and $36.67 \%$ regular knowledge about adequate food for DM. Regarding socioeconomic factors, 93.33\% of the participants have low family income and more than half (53.33\%) are illiterate. Of the 30 individuals studied, $43.33 \%$ are overweight and, although the same number of participants (43.33\%) are overweight, there is a prevalence of central adiposity in most individuals, based on the high values waist circumference (93.33\%) and waistto-hip ratio (83.33\%). Most of the patients in the study (76.66\%) had blood glucose above the recommendations for glycemic control of the Brazilian Diabetes Society. Conclusion: Despite the limitations, this study shows how socioeconomic factors can interfere in the knowledge about adequate nutrition for DM and, therefore, in the nutritional status and glycemic control of the participants. Thus, it is suggested to carry out more research in the region studied, with a greater number of participants and variables to corroborate the results found and provide more effective educational actions at the population level on the importance of adequate food as an essential part of treatment.

Keywords: Diabetes Mellitus type 2. Diet. Nutritional status. Socioeconomic Factors. Blood Glucose. 


\section{INTRODUÇÃO}

As doenças crônicas são caracterizadas por início gradual, de prognóstico usualmente incerto e com longa ou indefinida duração (BRASIL, 2012). Dentre essas doenças, Diabetes Mellitus (DM) é uma das mais estudadas e vem aumentando sua prevalência nos últimos anos por consequência da rápida urbanização, transição epidemiológica, transição nutricional, estilo de vida sedentário, excesso de peso, crescimento e envelhecimento populacional, além de maior sobrevida dos indivíduos com diabetes (SBD, 2019).

Segundo a Associação Americana de Diabetes (ADA, 2020), DM é um grupo de doenças metabólicas caracterizadas por hiperglicemia resultante de defeitos na secreção e/ou ação de insulina. A sua classificação é realizada através da etiologia, existindo o DM tipo 1 (DM1), DM tipo 2 (DM2), outros tipos específicos de DM e DM gestacional. Existem também duas categorias conhecidas como pré-diabetes: tolerância à glicose diminuída e a glicemia de jejum alterada, estas duas, assim como DM, apresentam riscos para doenças cardiovasculares (SBD, 2019).

DM, tanto do tipo 1 como do tipo 2, são doenças heterogêneas nas quais a apresentação clínica e sua progressão podem variar consideravelmente (ADA, 2020). O DM2 abrange pacientes que têm deficiência não absoluta de insulina e resistência periférica a esse hormônio. Esses indivíduos normalmente não necessitam de tratamento com insulina exógena para sobreviver e apresentam sobrepeso ou obesidade (HENNING, 2018).

O aumento da prevalência de DM no Brasil é possível ser percebido na Pesquisa Nacional de Saúde, desenvolvida pelo Instituto Brasileiro de Geografia e Estatística (IBGE) em 2013, a qual estimou que no Brasil, 6,2\% da população com 18 anos de idade ou mais referiu diagnóstico médico de diabetes (IBGE, 2014).

É comum que os indivíduos portadores de DM2 apresentem, além do sobrepeso ou obesidade, hipertensão, sedentarismo e maus hábitos alimentares. Uma alimentação rica em carboidratos simples, como a frutose, alimentos ricos em 
gorduras saturadas e trans, alimentos com alto índice glicêmico e carga glicêmica prejudicam o quadro clínico de um paciente com diabetes (VIA; MECHANICK, 2016).

Entretanto, apesar da alta prevalência de diagnóstico de DM, um estudo de Muninarayana e colaboradores (2010), realizado em zona rural, mostrou que 49,2\% dos 311 adultos entrevistados não sabiam o que era diabetes, revelando que existe ainda um grande desconhecimento sobre esse problema de saúde. Nessa mesma perspectiva, uma pesquisa sobre o conhecimento e prevenção do DM demonstrou que as pessoas com nível de escolaridade alto apresentam maior conhecimento a respeito da doença do que indivíduos com nível de escolaridade mais baixo (Wolde et al., 2017).

É imprescindível para o controle dessa enfermidade a manutenção de hábito de vida saudável, com promoção de uma dieta equilibrada, com teor de carboidratos controlado, bom aporte de fibras através do consumo de frutas e vegetais, utilização de alimentos de baixo índice glicêmico, com o intuito de evitar o descontrole glicêmico e complicações da doença (VIA; MECHANICK, 2016).

É fundamental para os pacientes com DM aprender formas de prevenção e também terem mais conscientização sobre o tratamento adequado (WOLDE et al., 2017). Pesquisas mostram que intervenções mais eficientes ocorrem a nível da comunidade, quando de forma permanente ou mantida por longos períodos, com interferência constante na população coberta (MACHADO et al., 2016; PORTERFIELD et al., 2009).

Diante desse contexto, o objetivo desse trabalho foi avaliar se o nível de conhecimento do paciente portador do DM2 sobre a alimentação adequada para o tratamento da doença, bem como fatores socioeconômicos, têm relação com controle glicêmico e estado nutricional. Consequentemente, a hipótese do presente estudo foi que um nível baixo de conhecimento sobre a alimentação adequada, bem como, más condições socioeconômicas, têm relação com o descontrole glicêmico e sobrepeso e/ou obesidade nos pacientes com DM2. 


\section{METODOLOGIA}

Trata-se de uma pesquisa de campo de tipo exploratório e descritiva com delineamento transversal e abordagem quantitativa, a qual foi realizada com usuários cadastrados nas Unidades de Saúde da Família (USF) Cristo Rei e Luiz Macena Aragão, localizadas no município de Uiraúna, Paraíba.

A população do estudo foi composta por indivíduos que faziam parte dessas duas USFs, com diagnóstico de DM2 de ambos os sexos, sem restrição de raça, maiores de 45 anos, incluindo os que faziam uso de hipoglicemiantes e insulina exógena. A amostragem foi não probabilística por conveniência composta por 30 indivíduos frequentadores do Grupo de Hipertensos e Diabéticos realizado pela equipe das USF's (Hiperdia). Foram excluídos da pesquisa: gestantes, adultos acamados ou impossibilitados de serem medidos e pesados, indivíduos que possuíam câncer de pâncreas e glicogenoses (erros inatos do metabolismo) por interferir no metabolismo da glicose.

O presente estudo foi aprovado pelo Comitê de Ética em Pesquisa da Faculdade Santa Maria ( $n^{\circ}$ do parecer 2.592.102) (ANEXO) e conduzido com base nos requisitos e critérios prescritos pela Resolução n 466/2012, do Conselho Nacional de Saúde (CNS), a qual versa sobre a ética em pesquisa com seres humanos.

Para avaliação do conhecimento dos pacientes diabéticos sobre a alimentação adequada para controle do DM2, foi aplicado um questionário com 16 questões adaptado de Anunciação et al. (2012) sobre alimentos específicos e práticas alimentares no manejo do DM. Além disso, dados sobre condições socioecônomicas, como renda familiar e escolaridade, e prática de atividade física também foram coletados.

Foi realizada avaliação antropométrica para conhecimento do perfil nutricional dos participantes, sendo aferidos peso corporal, altura e circunferências de cintura e quadril. A partir dessas aferições, foi possível calcular o Índice de Massa Corporal (IMC) e a Razão cintura-quadril (RCQ), sendo índices importantes para classificação 
do estado nutricional (LIPSCHITZ, 1994) e caracterização da distribuição da gordura abdominal (WHO, 2008; BRAY, 1989), respectivamente.

O teste de glicemia capilar foi realizado com tiras diagnósticas e glicosímetro (On Call Plus) (SBD, 2019), sendo o indivíduo orientado com antecedência para que estivesse em jejum. Realizou-se o teste como já acontecia no dia do encontro com o grupo de Hiperdia, que ocorreu nas quartas-feiras da primeira semana de cada mês.

Os dados obtidos foram tabulados no programa Microsoft Office Excel® 2016 para a realização da estatística descritiva e apresentados em percentuais, média e desvio-padrão A análise estatística foi realizada pelo software Sigmastat 3.5. Para interpretação dos dados, foram utilizadas tabelas e gráficos de frequências relativas para avaliação das condições socioeconômicas, estado nutricional e controle glicêmico.

Para avaliação do conhecimento dos participantes, foram consideradas duas categorias de conhecimento: menos da metade $(\leq 50 \%)$ e mais da metade de acertos das questões do teste, baseando-se nas escalas adotadas por Rothman et al. (2005), que considera baixo conhecimento um total de acertos $\leq 50 \%$ das questões e por Almeida et al. (1995), que considera conhecimento suficiente do assunto um percentual de acertos $\geq 70 \%$. Portanto, o percentual de acertos entre 51 e $69 \%$ foi considerado como conhecimento regular.

\section{RESULTADOS E DISCUSSÃO}

A presente pesquisa foi realizada no dia do encontro com o grupo de Hiperdia, no intuito de avaliar o nível de conhecimento relacionado a alimentação de indivíduos com DM2 através de questionários. Foram aplicados um total de 30 questionários, 15 na USF Cristo Rei e 15 na USF Luiz Macena Aragão. Os participantes do estudo apresentaram idade média de 69,77 \pm 9,60 anos, sendo $63,33 \%$ mulheres e $36,67 \%$ homens.

O questionário para avaliar o nível de conhecimento dos pacientes diabético apresentava 16 afirmações simples sobre a alimentação adequada para esse 
público, apenas para o participante assinalar "V" para as verdadeiras e "F" para as afirmações falsas. A partir da análise dos 30 questionários aplicados, obteve-se uma média de acertos de 8,50 $\pm 2,42$ questões, mostrando que os participantes acertaram em média aproximadamente apenas a metade das questões. Em relação à frequência do número de acertos, a partir da Tabela 1 pode-se perceber que metade dos participantes (50\%) apresentaram baixo conhecimento e $36,67 \%$ conhecimento regular. Com isso, apenas 4 participantes $(13,33 \%)$ mostraram ter conhecimento suficiente sobre práticas alimentares adequadas para o manejo do DM.

Tabela 1 - Frequência no questionário do número de acertos dos participantes.

\begin{tabular}{lc}
\hline Quantidade de acertos & Frequência \\
\hline$\leq 8$ acertos (Baixo conhecimento) & $50 \%$ \\
Entre 9 e 11 acertos (conhecimento regular) & $36,67 \%$ \\
$\geq 12$ acertos (conhecimento suficiente) & $13,33 \%$
\end{tabular}

Fonte: Próprio autor (2020).

A partir da Tabela 2, é possível observar que a maioria dos participantes era de baixa renda, pois 93,33\% tinham renda familiar de 1 a 2 salários mínimos. Alguns estudos já mostraram que a renda familiar tem influência na adesão de dietas para diabéticos, pois o planejamento de um plano alimentar adequado, composto por uma diversidade de nutrientes e fibras, é considerado dispendioso pelos pacientes com DM2 (ZANETTI et al., 2015; GIL et al., 2008; GRILLO et al., 2007).

Tabela 2 - Frequência dos fatores socioeconômicos dos participantes da pesquisa.

\begin{tabular}{lc}
\hline FATORES SÓCIOEOCNÔMICOS & Frequência \\
\hline Escolaridade & $53,33 \%$ \\
Analfabeto & $40,00 \%$ \\
Ensino fundamental incompleto & $0,00 \%$ \\
Ensino fundamental completo & $0,00 \%$ \\
Ensino médio incompleto & $6,66 \%$ \\
Ensino médio completo & \\
\hline Renda Familiar & $3,33 \%$ \\
Menos de 1 salário mínimo & $93,33 \%$ \\
De 1 a 2 salários mínimo & $3,33 \%$ \\
Mais de 3 salários mínimo & \\
\hline
\end{tabular}

Fonte: Próprio autor (2020). 
Quanto ao grau de escolaridade, também é possível ver na Tabela 2 que $53,33 \%$ das pessoas que participaram do estudo são analfabetas e, esses indivíduos, foram as que mais erraram as questões do questionário sobre conhecimento da alimentação e estilo de vida para o controle da doença, quando comparado às pessoas que tinham ensino médio incompleto $(40 \%)$ e o ensino médio completo $(6,67 \%)$.

Esses achados corroboram com outras pesquisas que relacionaram maior conhecimento sobre o DM com melhor grau de escolaridade, principalmente pessoas com educação com nível superior (MAINA et al., 2010). O nível de escolaridade é um fator interessante para ser analisado, pois a educação é um dos fatores principais para obtenção de um melhor entendimento sobre o controle e prevenção do DM (WOLDE et al., 2017). Portanto, é provável que o baixo nível de escolaridade encontrado na presente pesquisa tenha relação com o baixo conhecimento dos participantes sobre práticas alimentares adequadas no tratamento do DM.

Segundo Maina e colaboradores (2010), os indivíduos com diabetes até possuem algum conhecimento do que deve e do que não deve ser consumido, além do estilo de vida que deve ser adotado. Na realização dessa pesquisa, pode-se perceber que metade dos entrevistados tinha algum conhecimento sobre alimentação adequada para a doença, fato observado a partir da frequência de participantes que apresentavam conhecimento regular $(36,67 \%)$ ou suficiente $(13,33 \%)$ sobre alimentação adequada para DM. Entretanto, segundo Zanetti e colaboradores (2015), muitos não põem em prática os hábitos saudáveis por descuido ou por pensar que a alimentação não é acessível para sua renda familiar.

A partir da Figura 1, pode-se observar o estado nutricional dos participantes, dos quais 43,33\% estão acima do peso, constatado através do IMC superior a 27 $\mathrm{Kg} / \mathrm{m}^{2}$ (LIPSCHITZ, 1994). Apesar de também 43,33\% estarem com o peso adequado, nota-se prevalência de adiposidade central na maioria dos participantes, como pode ser observado com os altos valores de circunferência de cintura (93,33\%), a partir da classificação para o risco de doenças cardiovasculares (WHO, 2008), e razão cintura-quadril (83,33\%), através da classificação proposta por Bray 
(1989). Quanto à atividade física, fator relacionado ao estilo de vida importante para controle do DM2, 93,33\% dos indivíduos eram sedentários e o restante praticava apenas atividade leve (Figura 1).

Figura 1 - Frequência e classificação do estado nutricional, estilo de vida e glicemia em jejum dos participantes da pesquisa.

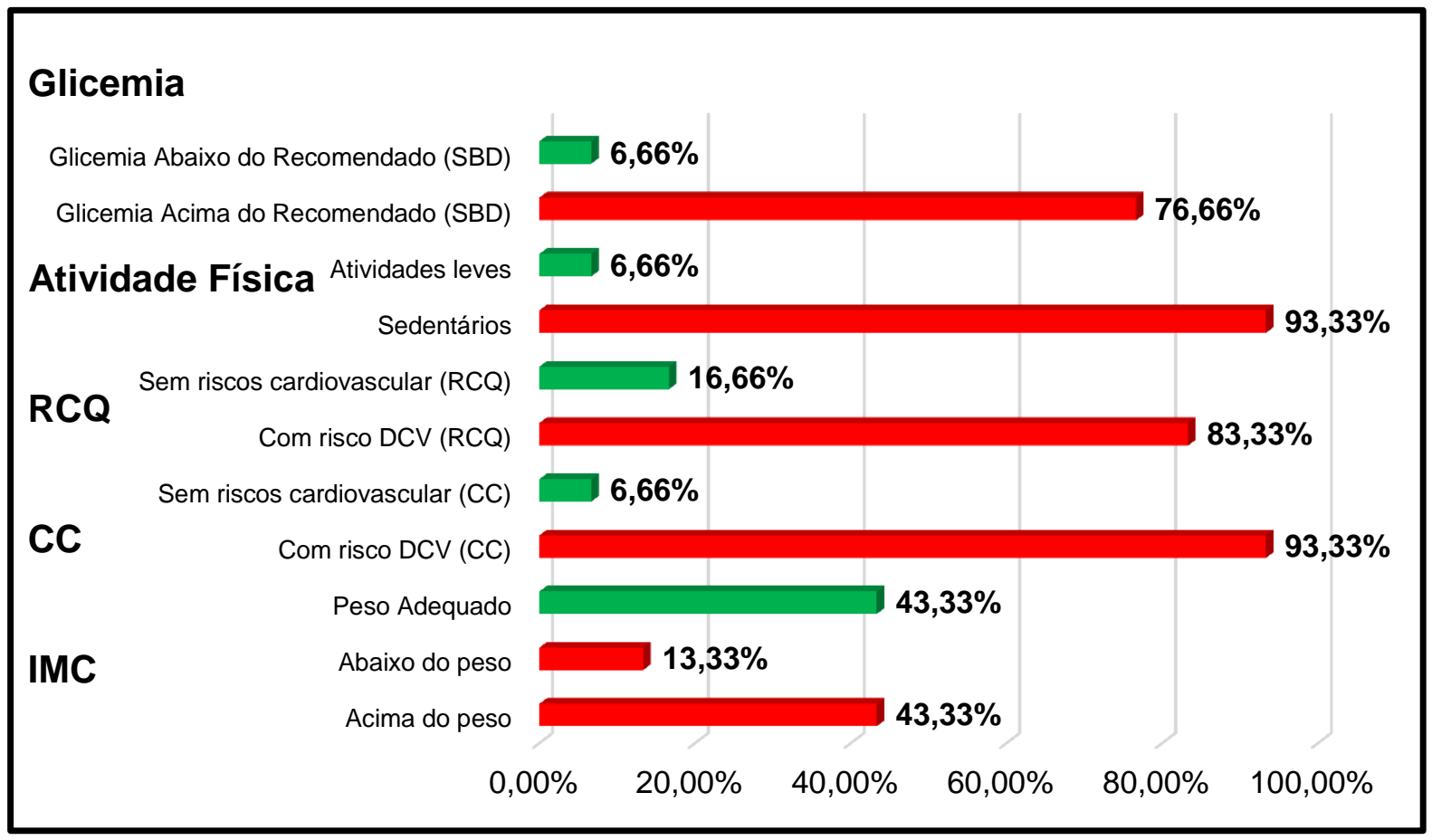

Fonte: Próprio autor (2020).

$\mathrm{CC}$ = circunfrência de cintura; $\mathrm{RCQ}=$ Razão cintura-quadril; IMC = Índice de massa corporal; DCV = doenças cardiovasculares; SBD = Sociedade Brasileira de Diabetes.

A recomendação da Sociedade Brasileira de Diabetes (SBD) para o controle de glicemia do paciente diabético é que ele mantenha sua glicose sérica em jejum em torno de $70-130 \mathrm{mg} / \mathrm{dL}$ (SBD, 2019). Na presente pesquisa, a maioria dos participantes (76,66\%) possui a glicemia maior do que $130 \mathrm{mg} / \mathrm{dL}$ (Figura 1), mostrando que não há o devido controle glicêmico.

Uma alimentação inadequada, rica em gorduras saturadas, alto índice glicêmico e carga glicêmica, baixo consumo de alimentos com fibras, o excesso de açúcares e sal na dieta; associada ao sedentarismo, contribuem para o aumento da 
obesidade e, consequentemente, o descontrole glicêmico (VIA; MECHANICK, 2016). Apesar de não ter sido feito o controle da dieta dos participantes no presente estudo, a falta de conhecimento sobre a alimentação adequada para doença, entre outros fatores associados, pode ter relação com o estado nutricional encontrado e, consequentemente, com o descontrole glicêmico encontrado.

A falta de atividade física e obesidade são fatores que contribuem para um baixo controle glicêmico dos pacientes com DM2, pois o sedentarismo auxilia no processo de depósito de gordura abdominal que induz significativamente a resistência à insulina (FIROUZI et al., 2015; KAVOURAS et al., 2007).

Para o tratamento do DM2, aderir à uma alimentação adequada e estilo de vida saudável, com realização de atividades físicas e controle do estado nutricional, é imprescindível para um bom controle glicêmico, e, portanto, diminuição de complicações, principalmente de doenças cardiovasculares que são as principais causas de morte de pacientes diabéticos (KUCUKARSLAN et al., 2009).

Em geral, foi observado que o conhecimento do paciente portador do DM2 sobre a alimentação adequada pode ter forte relação com a escolaridade. Tal conhecimento pode interferir no estado nutricional, o qual influencia diretamente o controle glicêmico, como já discutido. Assim, pode-se perceber que um bom manejo do DM2 envolve vários fatores que devem ser levados em consideração, com o intuito de planejar estratégias seguras e eficazes, melhorando a qualidade de vida dos pacientes (ASSUNÇÃO et al., 2017).

\section{CONCLUSÃO}

A partir dos resultados obtidos, foi possível observar o predomínio de baixas condições socioeconômicas e baixos níveis de atividade física e conhecimento sobre alimentação adequada na amostra avaliada. Esses fatores podem estar envolvidos com a prevalência de adiposidade central e, consequentemente, com o descontrole glicêmico apresentado pelos participantes da presente pesquisa. Os dados encontrados estão diretamente relacionados à qualidade de vida do portador de 
DM2, podendo aumentar o risco de morbidades, principalmente de doenças cardiovasculares.

Apesar da amostra pequena e de limitações, como a falta de controle das doses dos medicamentos e do consumo alimentar, esse estudo mostra como o grau de escolaridade e renda podem interferir no conhecimento sobre alimentação adequada para o DM e, por conseguinte, no estado nutricional e controle glicêmico dos participantes.

Dessa forma, espera-se que o presente estudo contribua para a reflexão dos profissionais de saúde e gestores sobre a importância da educação em saúde na atenção às pessoas com DM. Com isso, sugere-se a realização de mais pesquisas na região estudada com maior número de participantes, além da avaliação de mais variáveis para corroborar com os resultados encontrados e proporcionar ações educativas a nível populacional mais eficazes sobre a importância da alimentação adequada como parte imprescindível do tratamento.

\section{REFERÊNCIAS BIBLIOGRÁFICAS}

AIMEIDA, H. G. G. et al. Avaliação dos conhecimentos teóricos dos diabéticos de um programa interdisciplinar. Revista Latino-Americana de Enfermagem, v. 3, n. 2, p. 145-64, 1995.

AMERICAN DIABETES ASSOCIATION. Standards of Medical Care in Diabetes - 2020. Diabetes Care, v. 43, Suppl. 1, p. 81-90, 2020.

ANUNCIAÇÃO, P. C. et al. Avaliação do conhecimento sobre alimentação antes e após intervenção nutricional entre diabéticos tipo 2. Revista Baiana de Saúde Pública, v. 36, n. 4, p.986-1001, 2012.

ASSUNÇÃO, S.C. et al. Conhecimento e atitude de pacientes com diabetes mellitus da Atenção Primária à Saúde. Escola Anna Nery Revista de Enfermagem, v. 21, n. 4, p. 1-7, 2017.

BRASIL. Ministério da Saúde. Secretaria de Atenção à Saúde. Departamento de Atenção Básica. Documento de diretrizes para o cuidado das pessoas com doenças crônicas nas Redes de Atenção à Saúde e nas linhas de cuidado prioritárias. - Brasília: Ministério da Saúde, 2012.

BRAY, G. A. Classification and evaluation of the obesities. The Medical Clinics of North America, v.73, p.161-184, 1989.

FIROUZI, S. et al. Nutritional status, glycemic control and its associated risk factors among a sample of type 2 diabetic individuals, a pilot study. Journal of Research in Medical Sciences, v. 20 , n. 1 , p. $40-46,2015$.

GIL, G. P. et al. Conhecimento sobre diabetes mellitus de pacientes atendidos em programa 
ambulatorial interdisciplinar de um hospital universitário público. Semina: Ciências Biológicas e da Saúde, v. 29, n. 2, p. 141-154, 2008.

GOMES, F. S; ANJOS, L. A; DE VASCONCELLOS, M. T. L. Antropometria como ferramenta de avaliação do estado nutricional coletivo de adolescentes Anthropometry as a 22 tool for assessing the nutritional status of adolescents. Revista de Nutrição, v. 23, n. 4, p. 591-605, 2010.

GRILLO, M. F. F et al. Caracterização de pessoas com Diabetes Mellitus Tipo 2. Revista Brasileira de Enfermagem, v. 60, n. 1, p. 49-54, 2007.

HENNING, R. J. Type-2 diabetes mellitus and cardiovascular disease. Future Cardiology, v.14, n. 6 , p. 491-509, 2018.

IBGE. Pesquisa Nacional de Saúde 2013: percepção do estado de saúde, estilos de vida e doenças crônicas. Brasil, grandes regiões e unidades da federação. Rio de Janeiro, IBGE, 2014, 180p.

KAVOURAS, S. A. et al. Physical activity, obesity status, and glycemic control: The ATTICA study. Medicine and Science in sports and exercise, v. 39, n. 4, p. 606-611, 2007.

KUCUKARSLAN, A. et al. The effect of combined resistance and home-based walking exercise in type 2 diabetes patients. International Jornal of Diabetes in Developing Countries, v. 29, n.4, p. 159-165, 2009.

LIPSCHITZ, D. A. Screening for Nutritional Status in the Elderly. Primary Care, [S.I.], v.21, n.1, p.55-67, 1994.

MACHADO, A. P. et al. Educational strategies for the prevention of diabetes, hypertension, and obesity. Revista da Associação Médica Brasileira, v. 62, n. 8, p. 800-808, 2016.

MAINA, W. K. et al. Knowledge, attitude and practices related to diabetes among community members in four provinces in Kenya: a cross-sectional study. Pan African Medical Journal, $p$. $1-10,2010$.

MUNINARAYANA, C. et al. Prevalence and awareness regarding diabetes mellitus in rural Tamaka, Kolar. International Journal of Diabetes in Developing Countries, v. 30, n. 1, p. 1821, 2010.

PORTERFIELD, D. S. et al. Assessing local health department performance in diabetes prevention and control - North Carolina, 2005. Preventing Chronic Disease, v. 6, n. 3, p. 1-12, 2009.

ROTHMAN, R. L. et al. The spoken knowledge in low literacy in diabetes scale. The Diabetes Educator, v. 31, n. 2, p. 215-224, 2005.

SAMPAIO, L. R. Avaliação Nutricional. Salvador: Editora da Universidade Federal da Bahia, 162 p. 9. 2012.

SOCIEDADE BRASILEIRA DE DIABETES (SBD). Diretrizes da Sociedade Brasileira de Diabetes 2019-2020. São Paulo: Clannad, 2019.

VIA, M. A; MECHANICK, J. I. Nutrition in Type 2 Diabetes and the Matabolic Syndrome. Medical Clinics of North America, v. 100, n. 6, p. 1285-1302, 2016.

WHO. World Health Organization. Waist Circumference and Waist-Hip Ratio Report of a WHO Expert Consultation. Geneva, v.8, n.11, 2008. 
WOLDE, M. et al. Knowledge and practice on prevention of diabetes mellitus among diabetes mellitus family members, in suburban cities in Ethiopia. BMC Research Notes, v. 551, n. 10, p.1-6, 2017.

ZANETTI, M.L. et al. Adesão às recomendações nutricionais e variáveis sociodemográficas em pacientes com diabetes mellitus. Revista da Escola de Enfermagem da USP, v. 49, n. 4, p.619-625, 2015. 


\section{ANEXO - PARECER DO COMITÊ DE ÉTICA EM PESQUISA}

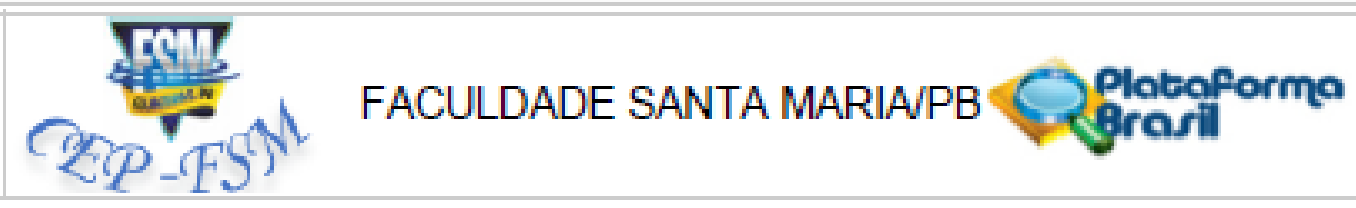

PARECER CONSUBSTANCIADO DO CEP

\section{DADOS DO PROJETO DE PESQUISA}

Titulo da Pesquisa: AVALIAÇÁ DO NIVEL DE CONHECIMENTO DO PACIENTE SOBRE A ALIMENTAÇÃO ADEQUADA PARA O TRATAMENTO DE DIABETES MELLITUS TIPO II

Pesquisador: LARISSA DE BRITO MEDEIROS

Área Tematica:

Versao: 2

CA.AE: 84369418.4 .0000 .5180

Institulçao Proponente: Faculdade Santa Maria/ FSM /PB

Patrocinador Princlpal: Financlamento Proprio

DADOS DO PARECER

Nùmero do Parecer: 2.592.102

Apresentaçåo do Projeto:

Trata-se de uma pesquisa de campo de tipo exploratorio e descritlva com dellneamento transversal e abordagem quantitativa. O local do estudo sera nas Unidades de Saude da Familla (USF) Cristo Rel e Lulz Macena Aragåo localizadas no municiplo de Ulraúna-PB. A populaçăo do estudo sera composta por Indlviduos moradores do municiplo de Ulraúna-PB que săo pacientes com dlagnostico de DM2 de ambos os sexos, sem restriçăo de

raça, malores de 45 anos, Incluindo os que fazem uso de hipogllcemlantes e Insulina exogena. A amostragem sera năo probablilstica por convenlencla composta por 50 indlviduos que fazem acompanhamento com a equipe das USF's frequentadoras do Grupo de Hipertensos e Dlabeticos reallzado pela equipe das USF's (Hiperdla).

Objetivo da Pesquisa:

Avallar o nivel de conhecimento do paclente com DM2 do municiplo de Uirauna-PB sobre a allmentaçăo adequada para sua doença e relaciona-la com controle gllcemico e estado nutricional.

Avallaçào dos Rlacos $\theta$ Beneficlos:

Os riscos e 05 beneficios foram adequadamente descritos, conforme preconizado na Resoluçăo 466/12, no TCLE e no arqulvo gerado na Plataforma Brasll.

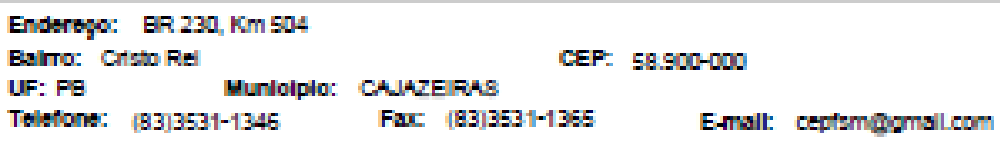




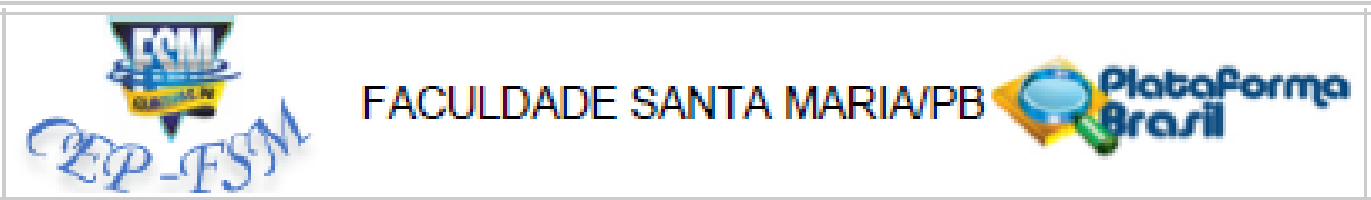

Contruaçso do Mareser: $2 \operatorname{sen} 2102$

Comentarios e Consideraçסes sobre a Pesqulsa:

A pesquisa esta bem dellneada e observa os preceltos eticos exigldos pela leglslaçăo, em especlal a Resoluçăo $466 / 12$.

Consideraçסি sobre 08 Termos de apresentaçăo obrigatorla:

Todos os Termos de apresentaçăo obrigatorla foram apresentados adequadamente: Termo de Consentimento Llvre e Esclarecido (TCLE); - Folha de rosto (datada e assinada); - Termo de Compromisso e responsabilldade do pesquisador responsavel (datado e assinado): Termo de Compromisso e responsabilldade do pesquisador participante (datado e assinado); - Projeto completo e Instrumento de coleta de dados.

\section{Recomendaç0өs:}

Atentar para envio do relatorio final ao CEP, conforme descrito na Resoluçăo 466/12 do Conselho Nacional de Saude.

Conclusoes ou Pendenclas e Llsta de Inadequaçoes:

Sem pendenclas elou Inadequaçbes.

Consideraç0өs Finals a criterio do CEP:

Este parecer fol elaborado baseado nos documentos abalxo relacionados:

\begin{tabular}{|c|c|c|c|c|}
\hline Tipo Documento & Arqulvo & Postagem & Autor & Sltuaçấo \\
\hline $\begin{array}{l}\text { Informaçdes Baslcas } \\
\text { do Proleto }\end{array}$ & $\begin{array}{l}\text { PB_INFORMAÇOES_BASICAS_DO_P } \\
\text { ROJETO 1087792.podf }\end{array}$ & $\begin{array}{c}25 / 03 / 2018 \\
17: 58: 37 \\
\end{array}$ & & Acelto \\
\hline Outros & novaanuencla.pdf & $\begin{array}{c}25 / 03 / 2018 \\
17: 58: 07\end{array}$ & $\begin{array}{l}\text { LARISSA DE BRITO } \\
\text { MEDEIROS }\end{array}$ & Acelto \\
\hline Folna de Rosto & FOLHADEROST01.pdf & $\begin{array}{c}05 / 03 / 2018 \\
18: 29: 19 \\
\end{array}$ & $\begin{array}{l}\text { LARISSA DE BRITO } \\
\text { MEDEIROS }\end{array}$ & Acelto \\
\hline $\begin{array}{l}\text { Projeto Detalhado / } \\
\text { Brochura } \\
\text { Investigador }\end{array}$ & $\begin{array}{l}\text { PROJETO_TCC_CLAUDIAFREITAS.do } \\
C X\end{array}$ & $\begin{array}{l}05 / 03 / 2018 \\
16: 18: 52\end{array}$ & $\begin{array}{l}\text { LARISSA DE BRITO } \\
\text { MEDEIROS }\end{array}$ & Acelto \\
\hline Outros & Questionario_conhecimento_DMil.pdi & $\begin{array}{c}05 / 03 / 2018 \\
16: 17: 24 \\
\end{array}$ & $\begin{array}{l}\text { LARISSA DE BRITO } \\
\text { MEDEIROS }\end{array}$ & Acelto \\
\hline Outros & QFCA.pdf & $\begin{array}{c}05 / 03 / 2018 \\
16: 16: 21\end{array}$ & $\begin{array}{l}\text { LARISSA DE BRITO } \\
\text { MEDEIROS }\end{array}$ & Acelto \\
\hline Outros & AN_ASE.pdf & $\begin{array}{l}05 / 03 / 2018 \\
16: 16: 02 \\
\end{array}$ & $\begin{array}{l}\text { LARISSA DE BRITO } \\
\text { MEDEIROS }\end{array}$ & Acelto \\
\hline $\begin{array}{l}\text { Declaraçăo de } \\
\text { Pesqulsadores }\end{array}$ & TCR_particlpante.pdf & $\begin{array}{l}05 / 03 / 2018 \\
16: 15: 32\end{array}$ & $\begin{array}{l}\text { LARISSA DE BRITO } \\
\text { MEDEIROS }\end{array}$ & Acelto \\
\hline $\begin{array}{l}\text { Declaraçắo de } \\
\text { Pesquisadores }\end{array}$ & TCR_responsavel.pdf & $\begin{array}{l}05 / 03 / 2018 \\
16: 15: 16\end{array}$ & $\begin{array}{l}\text { LARISSA DE BRITO } \\
\text { MEDEIROS }\end{array}$ & Acelto \\
\hline
\end{tabular}

Enderego: BR 230, $\mathrm{Km}$ 504 Boirro: Crtato Re

UF: PG

Uunloiplo: CAJAZERAS

Toletone: (B3)3531-1346
CEP: $58.900-000$

Fax $(83) 3531-1365$
E-malt: ceptemgomalicom 


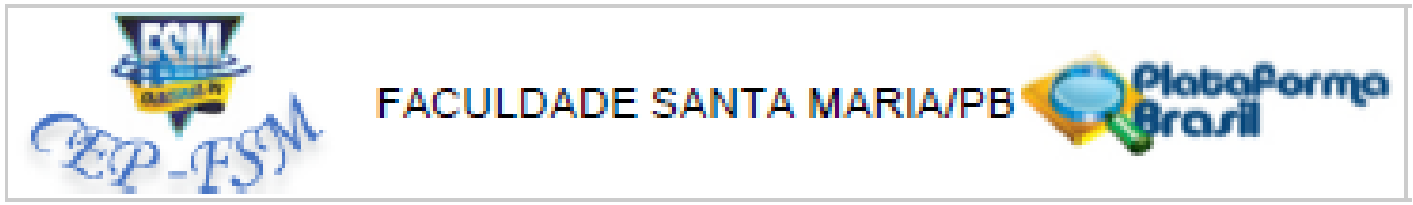

Conthusck do Pavear: 2.002 .102

\begin{tabular}{|c|c|c|c|c|}
\hline $\begin{array}{l}\text { TCLE/ Termos de } \\
\text { Assentimento / } \\
\text { Justifcativa de } \\
\text { Ausencla } \\
\end{array}$ & TCLE.pd" & $\begin{array}{c}05 / 03 / 2018 \\
16: 15: 04\end{array}$ & $\begin{array}{l}\text { LAFISSA DE BFITO } \\
\text { MEDEIROS }\end{array}$ & Acelto \\
\hline Orçamento & ORCAMENTO.Pd" & $\begin{array}{c}05 / 03 / 2018 \\
16: 14: 53\end{array}$ & $\begin{array}{l}\text { LAFISSA DE BRITO } \\
\text { MEDEIROS }\end{array}$ & Acelto \\
\hline Cronograma & CFONOGRAMA.PA & $\begin{array}{c}05 / 03 / 2018 \\
16: 14: 41\end{array}$ & $\begin{array}{l}\text { LAFISSA DE BRITO } \\
\text { MEDEIROS }\end{array}$ & Acelto \\
\hline
\end{tabular}

SItuaça do Parecer:

Aprovado

Necessita Apreclaça da CONEP:

Năم

CAJAZEIRAS, 10 de Abril de 2018

Assinado por:

ANKILMA DO NASCIMENTO ANDRADE

(Coordenador)

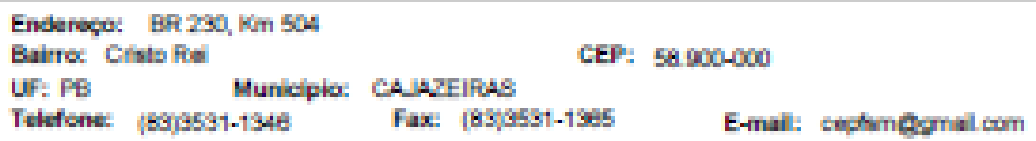

